\title{
Sulcus Vocalis: Our Experience
}

\author{
${ }^{1}$ Abhishek Gupta, ${ }^{2}$ Sukamal Das, ${ }^{3}$ Chandan Saha, ${ }^{4}$ Baisakhi Bakat, ${ }^{5}$ Soumitra Ghosh, ${ }^{6}$ Barin K Roychaudhuri
}

\section{ABSTRACT}

\section{Aims and objectives:}

- Evaluate the incidence of sulcus vocalis

- To document and analyze the outcome of treatment of sulcus vocalis.

Materials and methods: A prospective observational study, conducted over 1 year, at a tertiary care teaching hospital in the department of ENT and Head-Neck surgery. Patients attending a voice clinic with dysphonia were included in the study. The diagnosis was made by fiberoptic laryngoscopy (FOL) and confirmed by stroboscopy. Patients were classified into types I, II or III according to Ford classification (1996). Conservative management in the form of vocal hygiene and voice therapy was advocated for all patients. No improvement warranted surgery. Subjective voice handicap index-10 (VHI-10) and objective evaluation (FOL) was performed at diagnosis and at 3 and 6 months of follow-up. The minimum follow-up period was 6 months.

Results: A total of 167 patients attended the voice clinic for 6 months, of which 23 cases were diagnosed with sulcus vocalis. Of these 10 were females and 13 were males. 10 cases were categorized as type I, 9 as type II and 3 as type III. One patient had type II sulcus on right vocal fold and type I on the left side. All 10 cases of type I were treated satisfactorily by voice therapy. Out of 9 cases of Type II, 8 underwent microlaryngoscopic fat injection following voice therapy whereas 1 case was improved by voice therapy alone. In type III, patients 2 underwent fat implantation whereas 1 case underwent fat injection on one side and fat implantation on the other. All patients underwent post-operative voice therapy for 6 months. The reasonably good vocal outcome was noted during follow-up at 3 and 6 months.

Conclusion: Sulcus vocalis is not a very uncommon condition. Fibreoptic laryngoscopy aided with stroboscopy can confirm the

\footnotetext{
${ }^{1}$ Senior Resident, ${ }^{2} \mathrm{Head}$ and Neck Fellow, ${ }^{3}$ Speech and Language Pathologist, ${ }^{4}$ Fellow, ${ }^{5}$ Associate Professor, ${ }^{6}$ Senior Consultant and Surgeon

1,3,5,6 Department of ENT and Head Neck Surgery, Vivekananda Institute of Medical Sciences, Ramakrishna Mission Seva Pratishthan, Kolkata, West Bengal, India

${ }^{2}$ Department of ENT and Head Neck Surgery, Christian Medical College, Vellore, Tamil Nadu, India

${ }^{4}$ Department of ENT and Head Neck Surgery, Bombay Hospital, Mumbai, Maharashtra, India

Corresponding Author: Abhishek Gupta, Senior Resident, Department of ENT and Head Neck Surgery, Vivekananda Institute of Medical Sciences, Ramakrishna Mission Seva Pratishthan, Kolkata, West Bengal, India, e-mail: abhishekgupta. dr@gmail.com
}

diagnosis in all cases. Type I responds well to voice therapy. In case of type II and III sulcus, the reasonably good vocal outcome is achieved with surgical intervention, followed by prolonged voice therapy.

Keywords: Sulcus vocalis, Vocal fold, Voice therapy

How to cite this article: Gupta A, Das S, Saha C, Bakat B, Ghosh S, Roychaudhuri BK. Sulcus Vocalis: Our Experience. Int J Phonosurg Laryngol 2018;8(1):36-40.

\section{Source of support: Nil}

\section{Conflict of interest: None}

\section{INTRODUCTION}

The layered microstructure of vocal fold plays a key role in the production of normal voice. It consists of the epithelium, lamina propria, and the vocalis muscle. The superficial lamina propia (SLP) is composed of few elastic or collagenous fibers, which results in increased pliability of the vocal folds; the intermediate lamina propia is mainly composed of elastic fibers, and the deep lamina propia is composed of more collagenous fibers. The vocal ligament is formed by these elastic and collagenous fibres within the intermediate and deep layers. Below the deep lamina propia, the skeletal muscle fibers of the vocalis muscle form the deepest layer and body of the vocal folds. Any damage to this microstructure hampers phonation.

Sulcus vocalis is a depression or groove in the surface of vocal fold mucosa which is typically present on the leading edge of the vibratory surface. The mucosal cover is scarred down and tethered to the underlying vocal ligament along the varying length of the sulcus (Fig. 1).

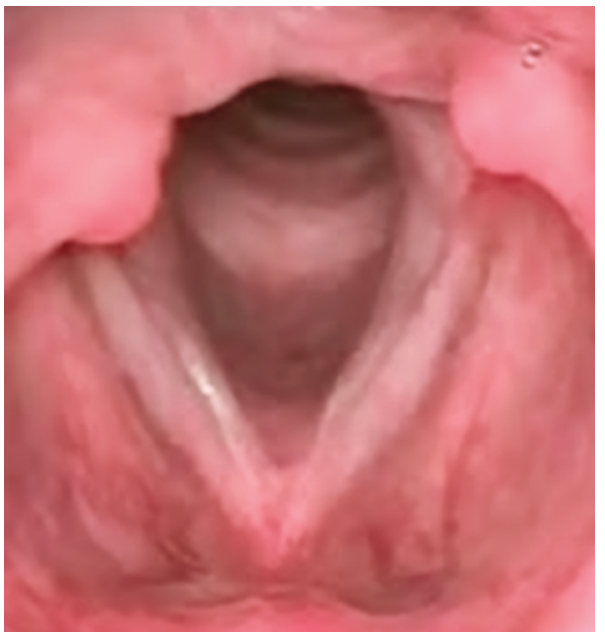

Fig.1: Laryngoscopy image of bilateral sulcus vocalis 
The incidence of sulcus vocalis is difficult to be determined because of the wide variation in presentation and diagnosis. Most sulci are undiagnosed because of subclinical symptoms, lack of clinician awareness, and difficulty in identification due to limited availability of laryngeal videostroboscopy. In a study on autopsy specimens by Nakayama et al., sulci were identified in $20 \%$ of specimens. ${ }^{1}$

Sulcus vocalis was classified into three categories by Ford et al. in $1996 .{ }^{2}$ In all these types the cleft was present on the medial surface of the vocal fold.

Ford Type I: The longitudinal depression of the vocal fold epithelium extends into the lamina propia, but does not reach the vocal ligament. Often this depression extends along the full length of the vocal fold. This variety is quite common in clinical practice and causes only a mild vocal dysfunction. Hence it is often termed as physiologic sulcus.

Ford Type II: The sulcus extends throughout the full length of the vocal cord. The depression extends up to the vocal ligament and there is also an associated loss of lamina propia disrupting the normal mucosal waves. This type has also been called sulcus vergeture.

Ford Type III: The lesion is deep and focal in nature appearing as a pit: the whole length being not involved. There is also an associated loss of lamina propia causing an affecting the mucosal wave. Histology of these lesions shows diffuse fibrosis, and inflammation.

There is a variant among these varieties known as the mucosal bridge. In this variety, there are two parallel sulci with normal appearing mucosa in between. It is challenging to treat this variety.

Patients may experience hoarseness but more often have symptoms of glottal insufficiency, including fatigue, poor volume, and poor projection. However, the voice may be normal with more subtle symptoms (e.g., fatigue, decreased vocal range with singing).

Sulcus vocalis is difficult to treat medically. Associated medical conditions affecting the voice like reflux laryngitis; allergic rhinitis should be evaluated and treated simultaneously. Before considering surgical therapy, all known sources of mechanical trauma are maximally reduced to determine reversibility and hopefully prevent a postoperative recurrence. This is accomplished in part by medical and voice therapy to reduce vocal trauma through improved phonatory techniques and vocal hygiene. Surgical options available for sulcus are implantation of autologous fat, fascia muscle etc. the fat can be injected either into paraglottic space to medialise the vocal fold or into the subepithelial space with an attempt to recreate the Reinke's space. Pulsed dye laser glottoplasty is also beneficial in the treatment of sulcus vocalis. ${ }^{3}$ Medialization thyroplasty has been advocated by some authors. An attempt to reconstitute the lamina propia may be considered for patients who have adequate volume but poor vocal quality. ${ }^{4}$

There appears to be a paucity of evidence-based algorithm for treatment of sulcus vocalis, and thus it requires identification and categorization of predictive clinical features that can further dictate the treatment. ${ }^{5}$

\section{AIMS AND OBJECTIVES}

- To evaluate the incidence of sulcus vocalis.

- To document and analyze the outcome of the treatment.

\section{MATERIALS AND METHODS}

A prospective observational study was conducted over a period of 1 year, at a tertiary care teaching hospital in the department of ENT and Head-Neck surgery. A total of 23 diagnosed adult patients of sulcus vocalis out of 167 patients who attended the voice clinic were studied. The study was accepted by the institutional ethical committee. Inclusion criteria: Adult (above the age of 18) patients of sulcus vocalis.

Exclusion criteria: Any associated lesions or growth in the larynx, neurological diseases of the larynx, children (below the age of 18 years), any patient with a history of previous surgery on vocal folds or larynx, and any prior history of trauma.

A detailed history of the patients attending the voice clinic was recorded in a preformed voice clinic proforma, following which a thorough ENT examination was performed in all of them. Initial diagnosis of sulcus vocalis was made with fiber FOL which was further confirmed by videostroboscopy. Patients were then classified in 3 categories I (Fig. 2), II (Fig. 3) and III (Fig. 4) according to the Ford classification (1996).

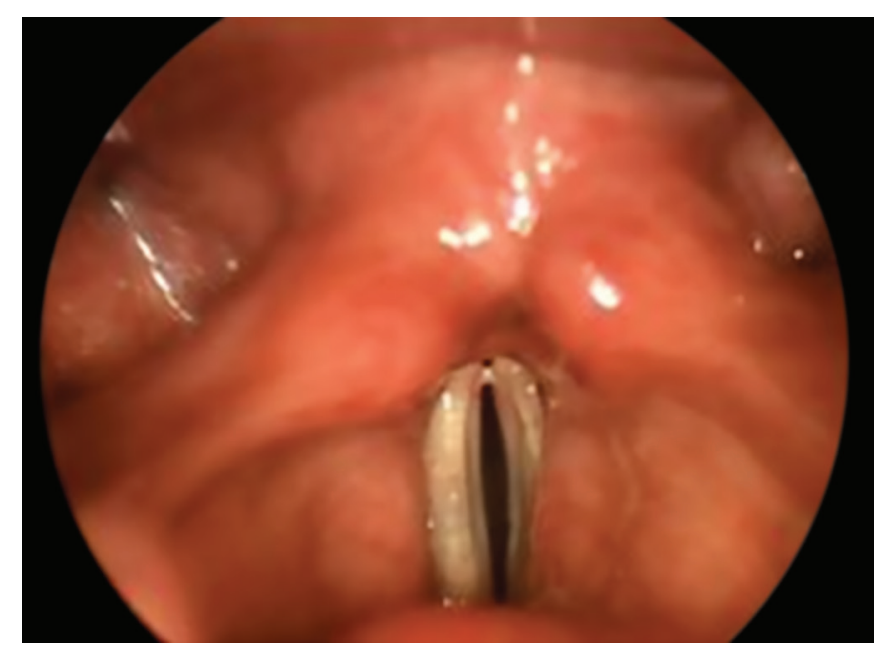

Fig. 2: Type I sulcus vocalis 


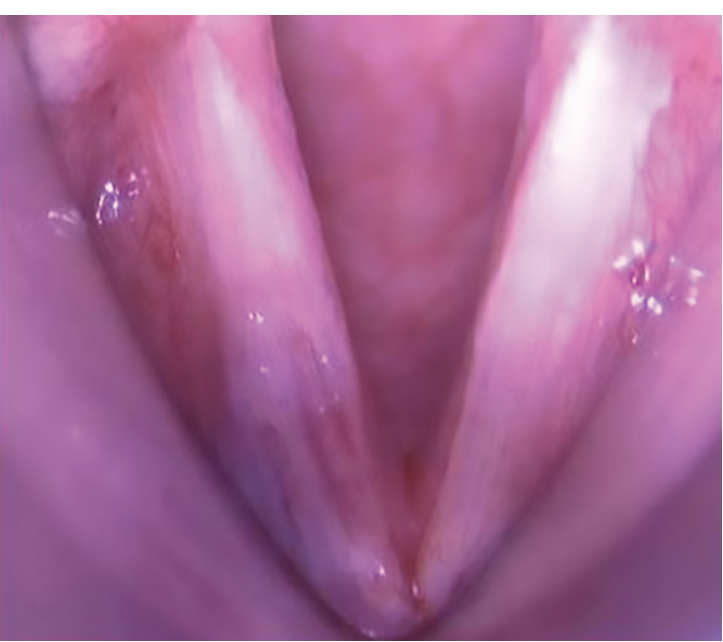

Fig. 3: Type II sulcus vocalis

Outcome measures were a subjective evaluation of voice based on Quality of Life (QOL) questionnaire, $\mathrm{VHI}-10$ and objective documentation of laryngeal finding by FOL. All the patients were asked to fill or were assisted to fill the VHI-10 for the subjective evaluation of their problem. A 3 weeks course of vocal hygiene and voice therapy was advised to all and a repeat FOL was performed at the end of 3 weeks. If dysphonia persisted with no symptomatic improvement or change in VHI, the patients were taken for microlaryngeal surgery under general anesthesia in the form of for either fat injection or fat implantation. Fat was harvested from the periumbilical region of the patient and minced with the help of small scissors to form a paste; it was then copiously irrigated with saline to wash out fatty acids, as they promote inflammation. This paste was kept in insulin to stabilize the adipocyte cell membrane. Thereafter it is loaded into a syringe attached to an 18 FG scalp vein set. It was then injected into the subepithelial space with one point of injection lateral to the sulcus. In the case of implantation, an incision was made lateral to the sulcus, and the sulcus elevated with micro dissector to create a pocket in which

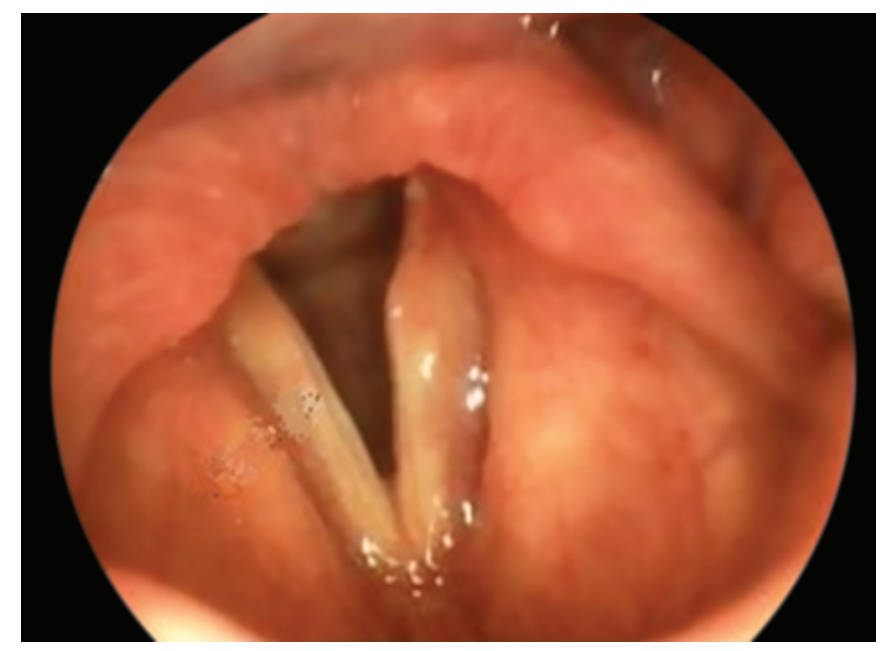

Fig. 4: Type III sulcus vocalis fat was implanted and secured with 5-0 Vicryl. Both techniques, overcorrection was made to compensate for any fat absorption subsequently. Vocal hygiene and voice therapy was given to all the patient's postoperatively for 6 months administered by qualified Speech and Language Pathologist trained in voice disorders. Subjective and Objective evaluation was performed using VHI-10 and FOL respectively at 3 and 6 months after surgery. The minimum follow-up period was 6 months.

\section{RESULTS}

A total of 167 patients attended the voice clinic with complaints of dysphonia. Out of 167 patients, 23 (14\%) were diagnosed with sulcus vocalis. Of these, $43 \%$ (10/23) were females and $57 \%(13 / 23)$ were males with a mean age of 41.1 years.

Of the 23 patients, 7 had unilateral disease whereas 16 had sulcus bilaterally.

Categorization of the type of sulcus was as follows (Fig. 5)

Type I-10/23 (45\%)

Type II-9/23 (40\%)

Type III-3/23 (15\%)

Mixed-1/23 (Right side: type II; Left side: type III (1\%)

All the 10 cases of type 1 sulcus vocalis had transitioned to satisfactory voice by vocal hygiene and voice therapy alone. Out of the 9 cases of type II, 8 underwent microlaryngoscopic injection of fat under general anesthesia; whereas 1 patient's voice improved with voice therapy alone. In the type III sulcus group 2 patients out of 3 underwent microlaryngoscopic fat implantation under general anesthesia whereas 1 patient underwent fat injection on one side and fat implantation on the other. All the patients were advised vocal hygiene and voice therapy for 6 months postoperatively (Graph 1).

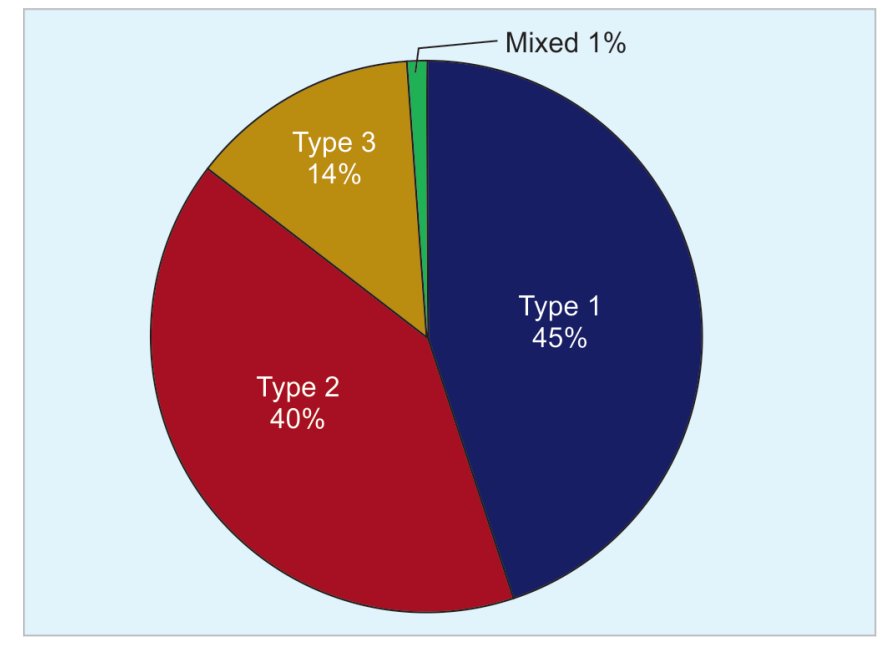

Fig. 5: Patients classified on the basis of the type of sulcus 


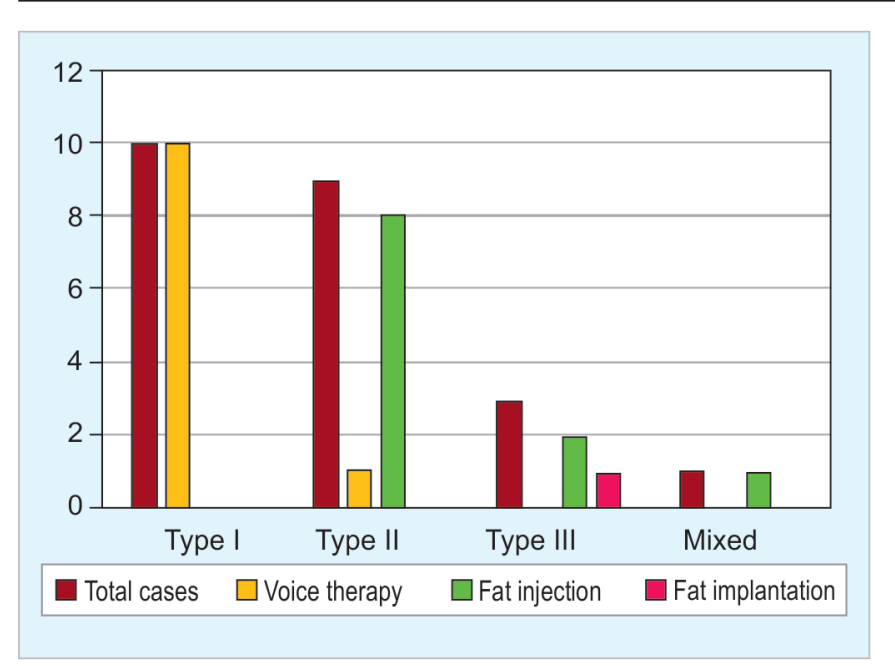

Graph 1: Treatment given

Subjective evaluation was done at 3 and 6 months after initiation of treatment for all 23 patients of the sulcus. All the patients had a reasonably good vocal outcome which was confirmed by the mean VHI (18.2 pretreatment and reduced to 1.7 posttreatment) (Graph 2). One patient of fat injection reported no improvement in voice quality.

Objective evaluation was also done by FOL at 6 months after initiation of treatment for all 23 patients of the sulcus. In 10 patients of type I sulcus, the finding of sulcus remained the same with correction of phonatory behavior, in spite of positive subjective improvement. Remaining 13 patients demonstrated healthy look of vocal folds with satisfactory phonatory closure except for one patient of type II sulcus (bilateral) who did not have voice improvement.

\section{DISCUSSION}

Sulcus vocalis is defined as a depression or groove in the surface of vocal fold mucosa, typically found on the leading edge of the vibratory surface. The mucosal cover, along with the sulcus, is scarred down to the underlying vocal ligament.

The incidence of sulcus vocalis is difficult to be determined, Nakayama et al. found sulci in $20 \%$ of autopsy specimens. ${ }^{1}$ In our study the incidence was found to be $14 \%$ out of 167 patients who attended voice clinic.

Welham et al. described sulcus vocalis as "a persistent challenge in this area as there is no evidence-based decision algorithm for treatment and thus it requires identification and categorization of predictive clinical features that can drive evidence-based treatment assignment. ${ }^{5}$

Rosen et al. concluded that maximum conservative therapy in the form of voice therapy should be tried before surgery. ${ }^{6}$ It was in agreement with our study as all type 1 cases and 1 case of type 2 case got resolved with voice therapy. Hence the importance of voice therapy in managing sulcus vocalis is beyond question.

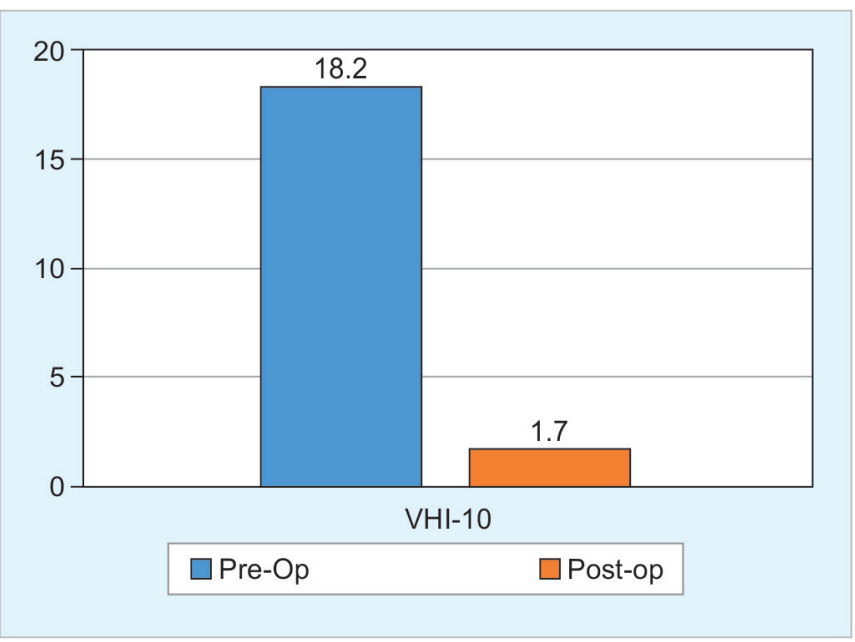

Graph 2: Mean VHI pre and post-operatively

Wen et al. 2004 suggested that the significance of accurate classification of sulcus vocalis which in turn is important for rightful treatment according to different types. ${ }^{7}$ Our results were in agreement with the above study.

Cantarella et al. suggested that autologous fat probably is the best augmentation material and is currently in widespread use. ${ }^{8}$ It is easily available, does not involve extra cost and works well; the only disadvantage being the absorption of fat by the body and thus requiring repeated injections. This absorption of fat can be minimized by meticulous preparation of fat and injecting or implanting of a greater volume than required. In our study fat was the only material used as well and none of the cases required repeat injections in 6 month follow-up period.

\section{CONCLUSION}

Sulcus vocalis is a fairly common condition. It may be diagnosed by fibreoptic laryngoscopy but needs to be confirmed by Videostroboscopy. Type I responds well to voice therapy, In the case of types II and III sulcus, the reasonably good vocal outcome is achieved with surgical intervention, followed by prolonged voice therapy. Hence, treatment should be tailor-made depending upon the type of sulcus to achieve the good phonatory outcome.

\section{CLINICAL SIGNIFICANCE}

The incidence of sulcus vocalis is relatively high amongst voice disordered patients. Though the diagnosis is simple, it can be missed unless a high index of suspicion is exercised. Categorization of the type of sulcus is important as it dictates the nature of treatment.

\section{LIMITATIONS OF THE STUDY}

Because of the small sample size, statistical significance cannot be calculated from this study. 


\section{REFERENCES}

1. Nakayama M, Ford CN, Brandenburg JH, et al. Sulcus vocalis in laryngeal cancer: a histopathologic study. Laryngoscope. 1994 Jan. 104(1 Pt 1):16-24.

2. Ford CN, Inagi K, Khidr A, Bless DM, Gilchrist KW. Sulcus Vocalis: A Rational Analytical Approach to Diagnosis and Management. Annals of Otology, Rhinology \& Laryngology. 1996, 105(3),189-200.

3. Hwang CS, Lee HJ, Ha JG, Cho CI, Kim NH, Hong HJ, et al. Use of pulsed dye laser in sulcus vocalis. Otolaryngology Head Neck Surgery. 2013 May;148(5):804-809.

4. Yilmaz T. Sulcus vocalis: excision, primary suture and medialization laryngoplasty: personal experience with
44 cases. Eur ArchOtorhinolaryngol. 2012;269(11):23812394 .

5. Welham NV1, Rousseau B, Ford CN, Bless DM. Tracking Outcomes After Phonosurgery For Sulcus Vocalis: A Case Report. J Voice. 2003 Dec;17(4):571-578.

6. Rosen CA. Vocal fold scar: evaluation and treatment. Otolaryngology Clinics of North America. 2000 Oct. 33(5):1081-1086.

7. Wen W1, Zhou SM, Zhang RX, Shen XH, Wang SM, Huang YD, et al. Diagnosis and management of sulcus vocalis. Zhonghua Er Bi Yan Hou Ke Za Zhi. 2004 Dec;39(12):733-736.

8. Cantarella G, Baracca G, Forti S, Gaffuri M, Mazzola RF. Outcomes of structural fat grafting for paralytic and nonparalytic dysphonia. Acta Otorhinolaryngol Ital. 2011Jun.31(3): 154-160. 